\title{
Adsorption of a directed polymer subject to an elongational force
}

\author{
E Orlandini ${ }^{1}$, M C Tesi $^{2}$ and S G Whittington ${ }^{3}$ \\ ${ }^{1}$ INFM, Dipartimento di Fisica, Università di Padova, Padova, Italy \\ 2 Dipartimento di Matematica, Università di Bologna, Bologna, Italy \\ ${ }^{3}$ Department of Chemistry, University of Toronto, Toronto M5S 3H6, Canada
}

Received 11 September 2003

Published 19 January 2004

Online at stacks.iop.org/JPhysA/37/1535 (DOI: 10.1088/0305-4470/37/5/005)

\begin{abstract}
We consider several different directed walk models of a homopolymer adsorbing at a surface when the polymer is subject to an elongational force which hinders the adsorption. We use combinatorial methods for analyzing how the critical temperature for adsorption depends on the magnitude of the applied force and show that the crossover exponent $\phi$ changes when a force is applied. We discuss the characteristics of the model needed to obtain a re-entrant phase diagram.
\end{abstract}

PACS numbers: $02.10 . \mathrm{Ox}, 05.40 . \mathrm{Fb}, 87.15 . \mathrm{Cc}$

\section{Introduction}

The use of optical tweezers (Svoboda and Block 1994, Ashkin 1997) has allowed the micromanipulation of polymer molecules. This has led to an interest in the unzipping of duplex DNA by an applied force (Essevaz-Roulet et al 1997). The temperature dependence of the critical force $f_{c}(T)$ required for unzipping has been studied for a variety of models and the phase diagram has been derived in the $\left(f_{c}, T\right)$-plane using exactly solvable models, scaling arguments and numerical approaches (Lubensky and Nelson 2000, Orlandini et al 2001, Marenduzzo et al 2001, 2002).

A simpler situation which is amenable to theoretical treatment, and which could also be investigated experimentally using similar micromanipulation techniques, is the adsorption of a polymer subject to an elongational force. We think of a linear polymer molecule attached at one end to an impenetrable surface at which the polymer can adsorb (i.e. there is an attractive interaction between the monomers and the surface). In addition, a force is applied to the other end of the polymer in a direction perpendicular to the surface. This applied force favours desorption and one expects a critical force, $f_{c}(T)$, for desorption which depends on the temperature $T$. At fixed $T$, if the force is less than $f_{c}(T)$ the polymer will be adsorbed, while if the force is larger than $f_{c}(T)$ the polymer will be desorbed. Hence the critical force 
curve $f_{c}(T)$ can be regarded as a phase boundary in the $(T, f)$-plane. In order to investigate the phase diagram (i.e. the form of $f_{c}(T)$ ) analytically we model the polymer by a directed walk either in two dimensions with the surface modelled by a line or in three dimensions with the surface modelled by a plane. We consider two different classes of directed walks in two dimensions, related to Dyck paths and to Motzkin paths, and use combinatorial methods for deriving the form of the grand partition function for each model. From this we derive the form of the phase diagram and calculate the values of the crossover exponent $\phi$ both with and without an applied force. We show that the exponent changes when a force is applied. We also consider a partially directed model in three dimensions and show that this model exhibits a re-entrant phase diagram.

In section 2, we consider adsorption of walks related to Dyck paths (see for instance, Janse van Rensburg 2000). With the geometry which we have chosen the walks can have at most half of their vertices in the adsorption line. In section 3 we remove this restriction by considering walks related to Motzkin paths (Janse van Rensburg 2000) and in section 4 we consider a partially directed model in three dimensions. Finally, we give some comments on our results in section 5 .

\section{Adsorption of walks related to Dyck paths}

We work in two dimensions (with coordinates $\left(x_{1}, x_{2}\right)$ ) and define $x_{2}=0$ as the line at which adsorption can occur. A Dyck path is a directed walk starting at the origin, with steps in the $(1,1)$ and $(1,-1)$ directions with the restrictions that (a) all vertices of the walk have non-negative $x_{2}$-coordinate and (b) the last vertex of the walk has zero $x_{2}$-coordinate. Let $d_{n}$ be the number of Dyck paths with $n$ edges, and we adopt the convention that $d_{0}=1$. Clearly $d_{2}=1, d_{4}=2, d_{6}=5$. If we define the generating function

$$
D(z)=\sum_{n} d_{n} z^{n}
$$

then Dyck paths can be factored according to the following scheme:

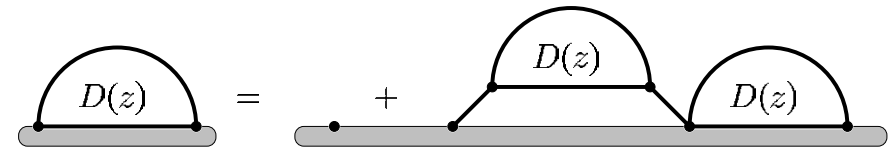

and hence $D(z)$ satisfies the recurrence relation

$$
D(z)=1+z^{2} D(z)^{2} .
$$

If we keep track of the number of vertices of the Dyck path with $x_{2}$-coordinate equal to zero, we can write $d_{n}(v)$ for the number of $n$-edge Dyck paths with $v+1$ vertices in the line $x_{2}=0$ and define the generating function

$$
D(x, z)=\sum_{v, n} d_{n}(v) x^{v} z^{n}
$$

Using the above factorization we have

$$
D(x, z)=1+x z^{2} D(1, z) D(x, z)
$$

so that

$$
D(x, z)=\frac{2}{2-x\left(1-\sqrt{1-4 z^{2}}\right)}
$$


We now consider a modification of the problem in which the final vertex of the walk does not necessarily have a zero $x_{2}$-coordinate. Let $b_{n}(v, h)$ be the number of these walks with $n$ edges, $v+1$ vertices in $x_{2}=0$ and whose last vertex has $x_{2}$-coordinate equal to $h$. Define the generating function

$$
B(x, y, z)=\sum_{v, h, n} b_{n}(v, h) x^{v} y^{h} z^{n} .
$$

Using a factorization argument similar to that used above, this generating function satisfies the relation

$$
B(x, y, z)=D(x, z)[1+y z B(1, y, z)]
$$

from which we obtain the result that

$$
B(x, y, z)=\frac{4 z}{\left(2-x+x \sqrt{1-4 z^{2}}\right)\left(2 z-y+y \sqrt{1-4 z^{2}}\right)} .
$$

This function has a square root singularity when $z=1 / 2$ and two other singularities when $2-x+x \sqrt{1-4 z^{2}}=0$ and when $2 z-y+y \sqrt{1-4 z^{2}}=0$. The square root singularity corresponds to the desorbed phase. The adsorption is controlled by whichever of the other two singularities is closest to the origin. We therefore solve each of these two equations for $z$ to find two critical surfaces

$$
z_{c}^{(1)}=\frac{y}{y^{2}+1}
$$

and

$$
z_{c}^{(2)}=\frac{\sqrt{x-1}}{x}
$$

The phase boundary in the $(x, y)$-plane is determined by the condition that $z_{c}^{(1)}=z_{c}^{(2)}$ which gives $y_{c}(x)=\sqrt{x-1}$. We can set $y=\mathrm{e}^{f / T}$ and $x=\mathrm{e}^{-\epsilon / T}$ where $f$ is the applied force, $\epsilon \leqslant 0$ is the interaction energy for a vertex in the interfacial line and $T$ is the temperature. This choice of $y$ is equivalent to setting the value of $h$ equal to zero when there is no applied force, and this is justified because the limiting free energy with no force, at constant $h$, is independent of $h$ for any finite $h$. Without loss of generality we set $\epsilon=-1$ so that $x=\mathrm{e}^{1 / T}$. With these substitutions we can plot the phase diagram in the $(T, f)$-plane and this is shown in figure 1 . When $f=0$ we have the usual adsorption transition at $x_{c}(0)=2$, i.e. $T_{c}(0)=1 / \log 2$. We can calculate the value of the crossover exponent $\phi$ from the behaviour of the limiting free energy in the adsorbed phase as the critical point is approached. Define $\beta=1 / T$. The limiting free energy $\kappa(\beta, f)$ is $-\log z_{c}(x, y)$ and $\phi$ is then given by (see for instance, Whittington 1998 or Janse van Rensburg 2000)

$$
1 / \phi=\lim _{\beta \rightarrow \beta_{c}(0)+} \frac{\log \left[\kappa(\beta, 0)-\kappa\left(\beta_{c}(0), 0\right)\right]}{\log \left[\beta-\beta_{c}(0)\right]} .
$$

A straightforward calculation shows that $\phi=1 / 2$ when $f=0$. A similar calculation when $f>0$ gives $\phi=1$ so that imposition of a force changes the nature of the adsorption singularity. We note that in the phase diagram in figure 1 the critical force is a monotonically decreasing function of temperature.

\section{Walks related to Motzkin paths}

A disadvantage with considering walks related to Dyck paths is that a maximum of half the vertices of the walk can lie in the line $x_{2}=0$. In this section, we generalize by considering 


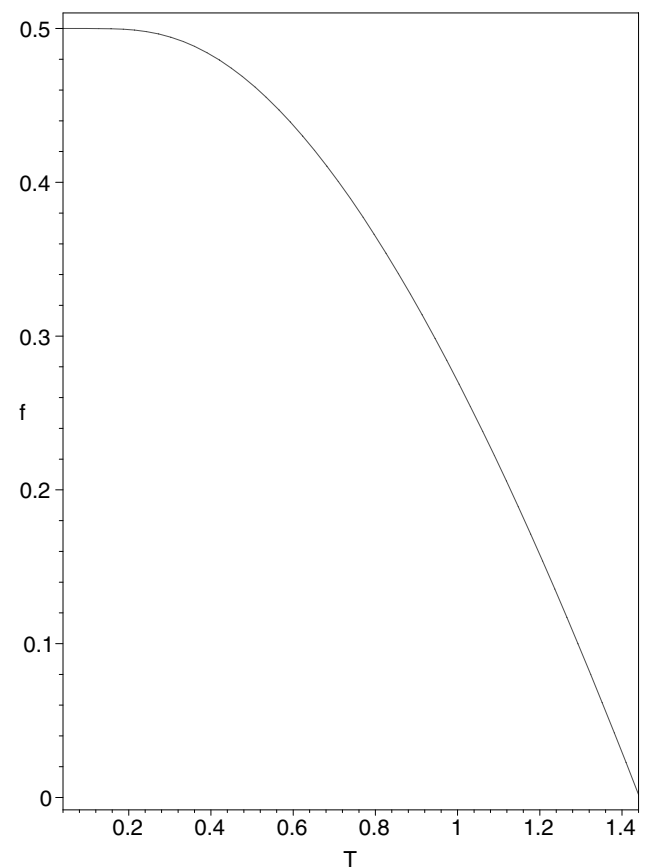

Figure 1. Force-temperature phase diagram for walks related to Dyck paths.

walks related to Motzkin paths. A Motzkin path is a directed walk starting at the origin with steps chosen from the set $\{(1,1),(1,-1),(1,0)\}$, with no vertex having negative $x_{2}$-coordinate and with the final vertex having zero $x_{2}$-coordinate. Let $l_{n}(v)$ be the number of Motzkin paths with $n$ edges and $v+1$ vertices in $x_{2}=0$. A similar factorization (to that leading to (2.2)) implies that the generating function

$$
L(x, z)=\sum_{v, n} l_{n}(v) x^{v} z^{n}
$$

satisfies the relation

$$
\begin{aligned}
L(x, z) & =\left(1+x z+x^{2} z^{2}+\cdots\right)\left(1+x z^{2} L(1, z) L(x, z)\right) \\
& =\frac{1+x z^{2} L(1, z) L(x, z)}{1-x z}
\end{aligned}
$$

This implies that

$$
L(x, z)=\frac{1}{1-z x-\frac{1}{2} x\left(-z+1-\sqrt{-3 z^{2}-2 z+1}\right)} .
$$

Relaxing the condition that the last vertex has zero $x_{2}$-coordinate, let $u_{n}(v, h)$ be the number of walks with $n$ edges, $v+1$ vertices in $x_{2}=0$ and whose last vertex has $x_{2}$-coordinate equal to $h$. Defining the generating function

$$
M(x, y, z)=\sum_{v, h, n} u_{n}(v, h) x^{v} y^{h} z^{n}
$$

we note (by another factorization argument) that $M$ satisfies the relation

$$
M(x, y, z)=L(x, z)[1+y z M(1, y, z)] .
$$




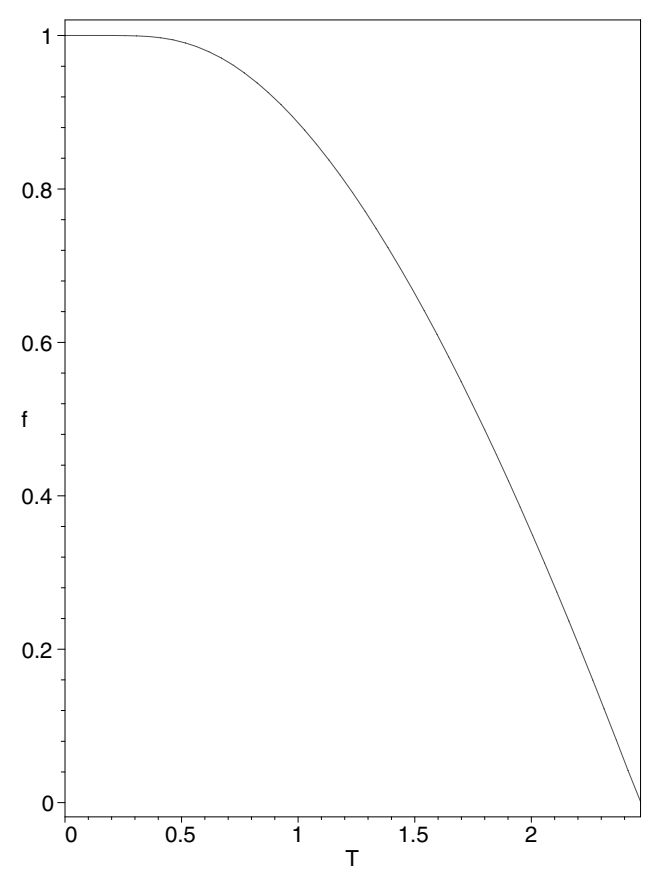

Figure 2. Force-temperature phase diagram for walks related to Motzkin paths.

Solving this gives

$$
M(x, y, z)=\frac{4 z}{(2-z x-x+x u)(2 z+y z-y+y u)}
$$

where $u=\sqrt{1-2 z-3 z^{2}}$. This expression for $M$ has a square root singularity when $z=1 / 3$ corresponding to the desorbed phase, and two singularities where the denominator is zero. Proceeding as for Dyck paths, we look for the values of $x$ and $y$ where the two singularities coincide and this gives the phase boundary in the $(x, y)$-plane. Making the substitutions $y=\mathrm{e}^{f / T}$ and $x=\mathrm{e}^{1 / T}$ we show the phase diagram in the $(f, T)$-plane in figure 2. Again we can calculate the crossover exponent $\phi$ when $f=0$, giving $\phi=1 / 2$. Similarly, when $f>0$ we obtain $\phi=1$, so that the nature of the adsorption transition changes when a force is applied. Again the force is a monotonically decreasing function of temperature.

As $T \rightarrow 0$, i.e. $x \rightarrow \infty, y_{c}(x) \sim x=\mathrm{e}^{1 / T}$ so that $f \rightarrow 1$. We note that the corresponding behaviour for the model discussed in section 2 is that $y_{c}(x) \sim \sqrt{x}$ and $f \rightarrow 1 / 2$. This reflects the fact that at most half of the vertices can be in the line $x_{2}=0$ for Dyck paths.

\section{Partially directed walks in three dimensions}

Both models considered in the previous sections have phase diagrams in which the force is monotone in the temperature so the phase diagrams are non-reentrant, while re-entrant phase diagrams have been seen in directed models of the denaturation of DNA by an applied force (Marenduzzo et al 2001, 2002). We have looked at a partially directed walk model in three dimensions to investigate the possibility of observing a re-entrant phase diagram in a model of polymer adsorption with an applied force. (A partially directed walk in two dimensions, 
interacting with a surface, has been considered by Owczarek and Prellberg (1993) though they were primarily interested in a different physical problem.)

The model which we consider is a self-avoiding walk in $Z^{3}$ with coordinates $\left(x_{1}, x_{2}, x_{3}\right)$. The walk starts at the origin and is confined to the half-space $x_{3} \geqslant 0$. The walk has no steps in the negative $x_{1}$ and negative $x_{2}$ directions. We index the walks by the number of vertices in the plane $x_{3}=0$.

We first consider walks with their last vertex in the plane $x_{3}=0$. If we write $g_{n}(v)$ for the number of such walks with $n$ edges and with $v+1$ vertices in $x_{3}=0$, we can define the generating function

$$
G(x, z)=\sum_{v, n} g_{n}(v) x^{v} z^{n} .
$$

This satisfies the recurrence relation

$$
\begin{aligned}
G(x, z)=(1+ & \left.2 x z+4 x^{2} z^{2}+\cdots+2^{p} x^{p} z^{p}+\cdots\right) \\
& \times\left[1+x z^{2}(G(1, z)-1)+2 x^{2} z^{3}(G(1, z)-1) G(x, z)\right]
\end{aligned}
$$

which can be written as

$$
G(x, z)=\frac{1+(G(1, z)-1) x z^{2}}{1-2 x z-2 x^{2} z^{3}(G(1, z)-1)} .
$$

The generating function of such walks with no interaction with the surface, $G(1, z)$, can be found by putting $x=1$ in (4.3). This gives

$$
G(1, z)=\frac{2 z^{3}-z^{2}-2 z+1-q}{4 z^{3}}
$$

where

$$
q=\sqrt{\left(2 z^{3}-z^{2}-2 z+1\right)^{2}-8 z^{3}\left(1-z^{2}\right)}
$$

$G$ has a square root singularity given by $q=0$, i.e.

$$
z^{*}=(\sqrt{17}-3) / 4
$$

In addition $G$ has a set of singularities corresponding to its denominator being zero, and this singular curve meets the square root singularity at

$$
\begin{aligned}
\left(x^{*}, z^{*}\right) & =\left(\frac{3-3 \sqrt{3}+\sqrt{17}(\sqrt{3}-1)}{13-3 \sqrt{17}}, \frac{\sqrt{17}-3}{4}\right) \\
& =(1.3036 \ldots, 0.2807 \ldots) .
\end{aligned}
$$

which corresponds to the location of the adsorption transition in the absence of an applied force.

In order to include a force term we allow the walks to have their last vertex with $x_{3}$ coordinate not equal to zero. Let $w_{n}(v, h)$ be the number of such walks with $n$ edges, $v+1$ vertices in $x_{3}=0$ and with the $x_{3}$-coordinate of the last vertex equal to $h$. Define the generating function

$$
W(x, y, z)=\sum_{v, h, n} w_{n}(v, h) x^{v} y^{h} z^{n}
$$

In order to compute $W$ it is convenient to consider the subset of walks contributing to $G$ characterized by having their last edge in the plane $x_{3}=0$. We define their generating function to be $F(x, z)$. Then by a factorization argument we can write

$$
F(x, z)=\left(1+2 x z+\cdots+2^{p} x^{p} z^{p}+\cdots\right)\left(1+2 x^{2} z \mathcal{L}(z) F(x, z)\right)
$$




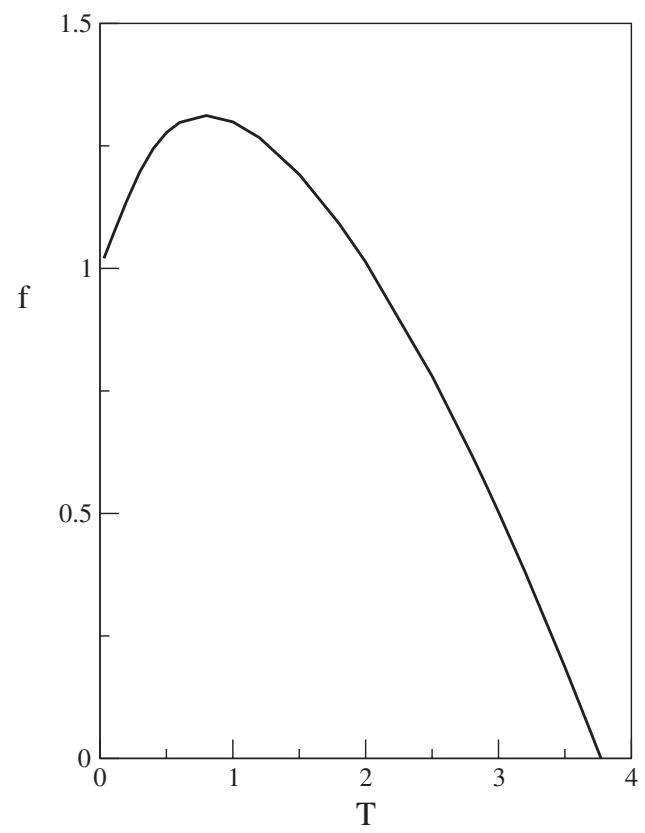

Figure 3. Force-temperature phase diagram for partially directed walks in three dimensions.

where $\mathcal{L}(z)=z^{2}[G(1, z)-1]$ is the generating function for walks with only the two vertices of degree 1 in $x_{3}=0$. This gives

$$
F(x, z)=\frac{1}{1-2 x z-2 x^{2} z \mathcal{L}(z)} .
$$

Then $W$ is given by the equation

$$
W(x, y, z)=G(x, z)+y z F(x, z) W(1, y, z)
$$

where the first term on the right-hand side comes from walks ending in $x_{3}=0$ and the second term is from walks leaving the surface to end in a half-space walk (or tail). By substituting $x=1$ we obtain an explicit expression for $W(1, y, z)$ and hence an expression for $W(x, y, z)$. $W$ is singular when $z=z^{*}$ and when the denominator of $W$ is zero. The denominator factors and we have two possible sets of zeros, i.e. when

$$
2-x^{2}-4 x z+2 x^{2} z+x^{2} z^{2}+2 x^{2} z^{3}+x^{2} q=0
$$

or when

$$
1-2 z-2 y z+z^{2}+2 z^{3}+q=0
$$

where $q$ is given by (4.5). We then define $z_{c}^{(1)}(x)$ to be a real positive root of (4.12) and $z_{c}^{(2)}(y)$ to be a real positive root of (4.13) and set $z_{c}^{(1)}=z_{c}^{(2)}$ to determine the phase boundary in the $(x, y)$-plane. With the changes of variable used in the two-dimensional case we then obtain the phase boundary in the $(T, f)$-plane, shown in figure 3. When $f=0$ we have the usual adsorption transition at $T_{c}(0)=1 / \log x^{*}$.

We note that the force goes through a maximum as the temperature varies so the phase diagram is re-entrant. We return to this point in the next section. 


\section{Discussion}

We first considered two directed walk models of polymer adsorption in two dimensions when the polymer is subject to an elongational force normal to the adsorbing line. We derived the form of the phase boundary in the force-temperature plane, i.e. the temperature dependence of the critical force for adsorption. As the temperature goes to zero, the critical force goes to a constant but the constant is $1 / 2$ for the Dyck path model and 1 for the Motzkin path model. This reflects the difference in the ground state energies of the two models. When the applied force is zero the crossover exponent characterizing adsorption is $1 / 2$, as found for other directed models of polymer adsorption (Whittington 1998, Janse van Rensburg 2000). This contrasts with the value $\phi=1$ when there is a positive force. This implies that the order of the transition changes when a force is applied.

These models are reminiscent of models which have been used to study the mechanical denaturation of duplex DNA (Marenduzzo et al 2001). Also in that case the order of the phase transition for directed models changes when the force is applied. The behaviour of the phase boundary in the adsorption and denaturation models is qualitatively similar except that for denaturation the phase boundary shows re-entrant behaviour-i.e. the critical force goes through a maximum as $T$ varies. It is believed that this may be associated with the fact that the model has an entropic contribution (which is extensive in $n$ ) in the zipped phase but not in the unzipped phase. Our models for adsorption in two dimensions have only a single configuration contributing at $T=0$, which corresponds to zero entropy at $T=0$. We have extended these models to a three-dimensional case where the system has residual entropy at $T=0$, and we showed that this model has a re-entrant phase diagram, consistent with the above interpretation.

We can understand the difference in the low temperature behaviour in the three models we have considered by the following simple and approximate argument. Suppose that $T$ is close to zero. One can think of the polymer as $m$ monomers adsorbed and $n-m$ desorbed under the applied force. The free energy of the $n$-mer can be approximately written as

$$
F_{n}=-f(n-m)-m \epsilon-m T \log \mu
$$

where $-m \epsilon$ is the energy associated with the $m$ adsorbed monomers, and $\log \mu$ is associated with the conformational entropy per monomer in the adsorbed state. Differentiating with respect to $m$ and setting the derivative equal to zero to find the critical force we obtain

$$
f_{c}(T)=\epsilon+T \log \mu .
$$

For the Dyck path model $\epsilon=1 / 2$ since only half the monomers can be in the surface, and $\mu=1$ since there is only one contributing conformation in the completely adsorbed state. For the Motzkin path model $\epsilon=1$ and $\mu=1$ while for the partially directed walk model in three dimensions $\epsilon=1$ and $\mu=2$, since there are $2^{n}$ conformations of the partially directed walk (with $n$ edges) when it lies completely in the adsorbing plane. Hence $f_{c}(0)$ is $1 / 2,1$ and 1 for the three models and $\mathrm{d} f_{c}(T) / \mathrm{d} T$, evaluated at $T=0$, is zero for the models in two dimensions but $\log 2$ for the three-dimensional model.

One could think of measuring the force needed for desorption and in principle the experiment could be carried out at different temperatures. It would be interesting to explore the re-entrant behaviour experimentally.

\section{Acknowledgments}

The authors wish to thank Professor T Cosgrove for useful discussions and NSERC for financial support. SGW would like to acknowledge the hospitality of the Newton Institute, Cambridge, where part of this work was carried out. 


\section{References}

Ashkin A 1997 Proc. Natl Acad. Sci. USA 94 4853-60

Essevaz-Roulet B, Bockelmann U and Heslot F 1997 Proc. Natl. Acad. Sci. USA $9411935-40$

Janse van Rensburg E J 2000 The Statistical Mechanics of Interacting Walks, Polygons, Animals and Vesicles (Oxford: Oxford University Press)

Lubensky D K and Nelson D R 2000 Phys. Rev. Lett. 85 1572-5

Marenduzzo D, Bhattacharjee S M, Maritan A, Orlandini E and Seno F 2002 Phys. Rev. Lett. 8802028102

Marenduzzo D, Trovato A and Maritan A 2001 Phys. Rev. E 64031901

Orlandini E, Bhattacharjee S M, Marenduzzo D, Maritan A and Seno F 2001 J. Phys. A: Math. Phys. 34 L751-L758

Owczarek A L and Prellberg T 1993 J. Stat. Phys. 70 1175-94

Svoboda K and Block S M 1994 Ann. Rev. Biophys. Biomol. Struct. 23247

Whittington S G 1998 J. Phys. A: Math. Gen. 31 8797-803 